\title{
A rare presentation of prostate cancer with diffuse osteolytic metastases and PSA of $7242 \mathrm{ng} / \mathrm{ml}$
}

\author{
Govarthanan Rajendiran, Linda Green, Gurdeep Chhabra
}

\begin{abstract}
Introduction: Bone metastases from prostate cancer are commonly osteoblastic and can result in disabling skeletal complications and affect quality of life. We report a rare case of prostate cancer presenting with diffuse osteolytic metastases. Case Report: A 65-yearold African American man, with no past medical history or specific urologic symptoms, sought medical attention for non-specific pain symptoms. He had metastatic prostate adenocarcinoma, high PSA level $(7242 \mathrm{ng} / \mathrm{mL})$ and extensive osteolytic metastases. After one year of therapy with antiandrogens, zoledronic acid and local radiation, patient was asymptomatic and did not suffer disabling skeletal complications. His PSA level has been stable and less than $1 \mathrm{ng} / \mathrm{mL}$. Conclusion: Prostate cancer can rarely present with diffuse osteolytic metastases. Early initiation of supportive treatment, especially the
\end{abstract}

Govarthanan Rajendiran', Linda Green², Gurdeep Chhabra $^{3}$

Affiliations: ${ }^{1}$ PGY-III Pesident/Chief resident, Dept. of Internal Medicine, Prince George's Hospital Center, Maryland, USA; ${ }^{2}$ Assistant program director, Dept. of Internal Medicine, Prince George's Hospital Center, Maryland, USA; ${ }^{3}$ Hemato-oncologist, Prince George's Hospital Center, Maryland, USA.

Corresponding Author: Govarthanan Rajendiran, 3001 Hospital Drive, J-500, $5^{\text {TH }}$ Floor, Department of Internal Medicine, Cheverly, Maryland, USA 20785; Ph: 1-(301) 768-3802; Fax: +1-(301) 618-2986; Email: govdocus@gmail.com

Received: 28 March 2011

Accepted: 18 August 2011

Published: 01 September 2011 bisphosphonate zoledronic acid, plays a pivotal role in the reduction of disabling skeletal complications.

Keywords: Prostate adenocarcinoma, Osteolytic metastases, Androgen deprivation therapy, Skeletal related event, Zoledronic acid

$* * * * * * * * *$

Rajendiran G, Green L, Chhabra G. A rare presentation of prostate cancer with diffuse osteolytic metastases and PSA of $7242 \mathrm{ng} / \mathrm{ml}$. International Journal of Case Reports and Images 2011;2(9):16-20.

$$
* * * * * * * * *
$$

doi:10.5348/ijcri-2011-09-55-CR-5

\section{INTRODUCTION}

Prostate cancer is the second leading cause of cancer-related death in the United States among men and is one of the most commonly diagnosed cancer in American males $[1,2]$. Most prostate cancer-related morbidity and mortality are due to advanced disease, when it can metastasize to various regions of the body, most commonly the lymph nodes, bone and lungs. The bones that are most commonly involved are vertebrae, sternum, pelvic bones, ribs, and femurs, and the characteristic radiologic appearance is osteoblastic but it can rarely present with predominantly osteolytic appearance [3]. In bone metastases, pathologic activation of both osteoblasts and osteoclasts are present and the dominance is influenced by the specific interaction of tumor with the bone environment. In fact, the markers of bone resorption are elevated in patients with bone metastases from prostate cancer to a greater degree than bone metastases from any other malignancies $[3,4]$. Hence 
prostate cancer has strong resorptive component in addition to the element of bone formation and this case profiles an advanced prostate cancer patient with predominant resorptive component resulting in extensive osteolytic metastases.

\section{CASE REPORT}

A 65-year-old African American man with no past medical history presented with intermittent left gluteal pain for three months. He did not have obstructive urinary symptoms, incontinence, back pain, weakness or numbness. He denied any physician evaluation or hospital visit in the last 35 years, as he did not suffer from any major illness and treated himself with overthe-counter medications for minor ailments. Examination findings were normal, except for an illdefined swelling in the left gluteal region and enlarged very hard prostate. His blood test results were normal; except for a highest PSA level of $7242 \mathrm{ng} / \mathrm{mL}$.

Computed tomography (CT) imaging of abdomen, pelvis and thorax showed diffuse osteolytic metastases involving the entire spine, ribs and pelvis, and there was an isolated expansile lytic lesion in the left iliac bone (Figures 1, 2). Magnetic resonance imaging (MRI) study of the entire spine showed similar findings but did not show any evidence of fracture or dislocation or significant cord compression (Figure 3). Whole body Technetium bone scan showed diffuse reduce uptake (cold spots) involving several ribs and vertebrae suggestive of extensive osteolytic activity, along with mild irregular uptake suggestive of minimal osteoblastic component (Figure 4).

Prostate biopsy revealed adenocarcinoma with a Gleason score of $(4+3=7)$ which is associated with unfavorable outcome. Serum protein electrophoresis with immunofixation showed a non-quantifiable $M$ spike. Patient was advised biopsy of the iliac bone lesion and bone marrow, but he refused. As he did not have any evidence of multiple myeloma or other malignancy, he was presumptively diagnosed to have metastatic prostate adenocarcinoma with diffuse osteolytic metastases. He was treated with bicalutamide, leuprolide and zoledronic acid (ZA), along with palliative radiotherapy to the left iliac bone lesion. He received 3500 cGy total dose radiation in 14 fractions (250 cGy dose per fraction) to the left superior pelvis and sacroiliac joint.

After one year of therapy, his PSA has normalized at $0.66 \mathrm{ng} / \mathrm{mL}$ and he was asymptomatic. Posttreatment CT scan of pelvis showed considerable regression of left iliac lytic bone lesion (Figure 5). MRI spine showed significant resolution of lytic lesions with mild residual lesions involving several ribs and vertebrae, and no new lesions were detected.

\section{DISCUSSION}

In the literature, there are very few reported cases

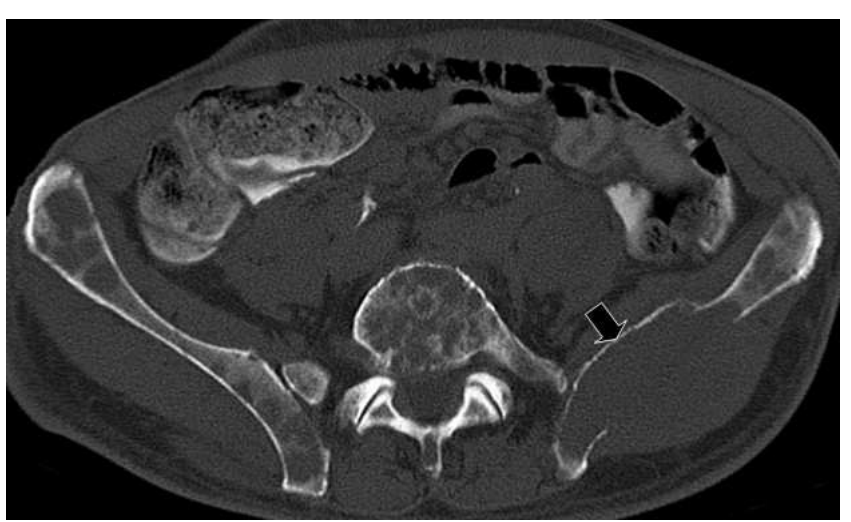

Figure 1: CT scan of the pelvis shows lytic lesion in the left iliac bone (arrow).

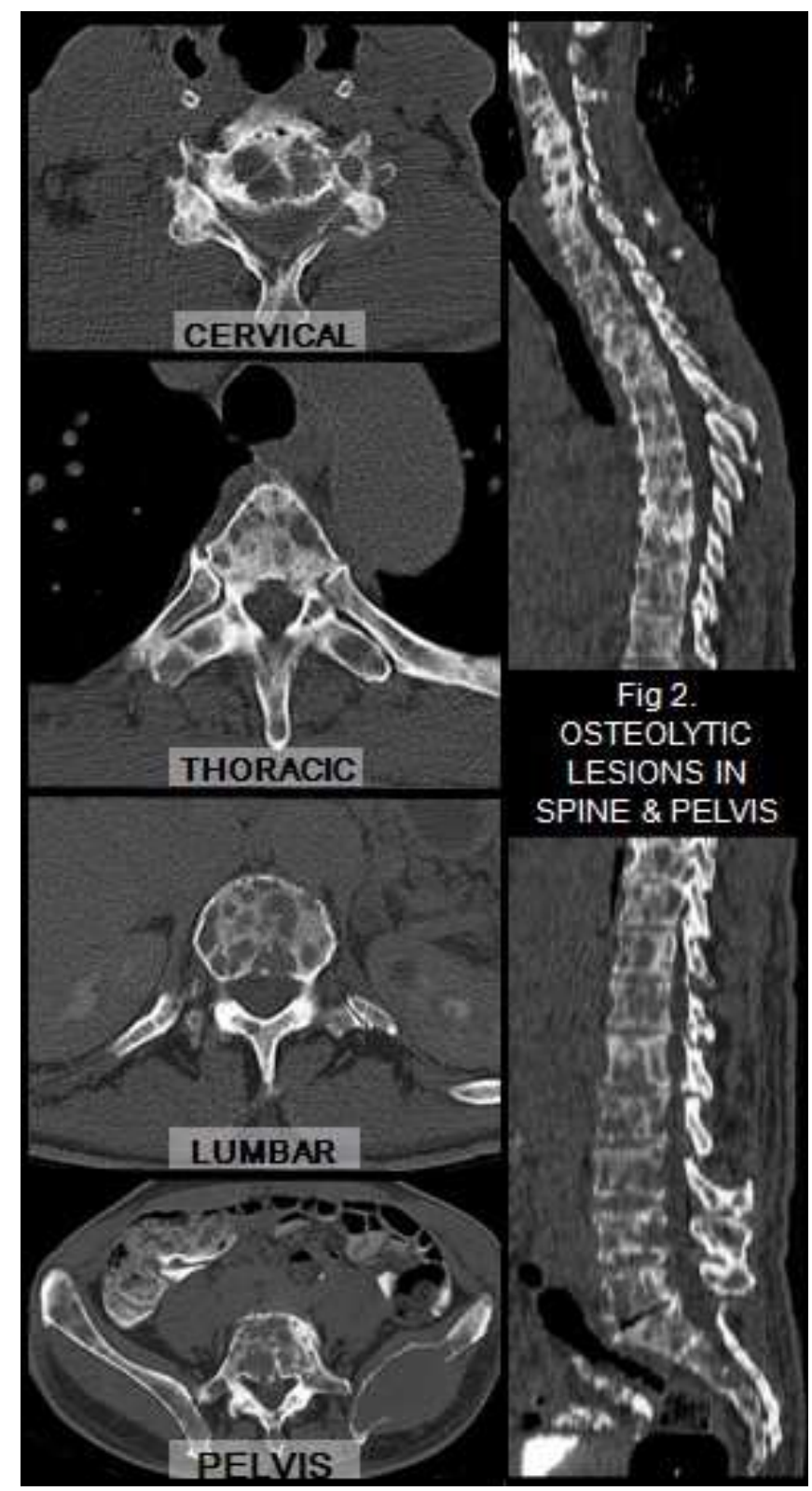

Figure 2: Non-contrast CT scan of the entire spine shows diffuse multiple osteolytic lesions. 


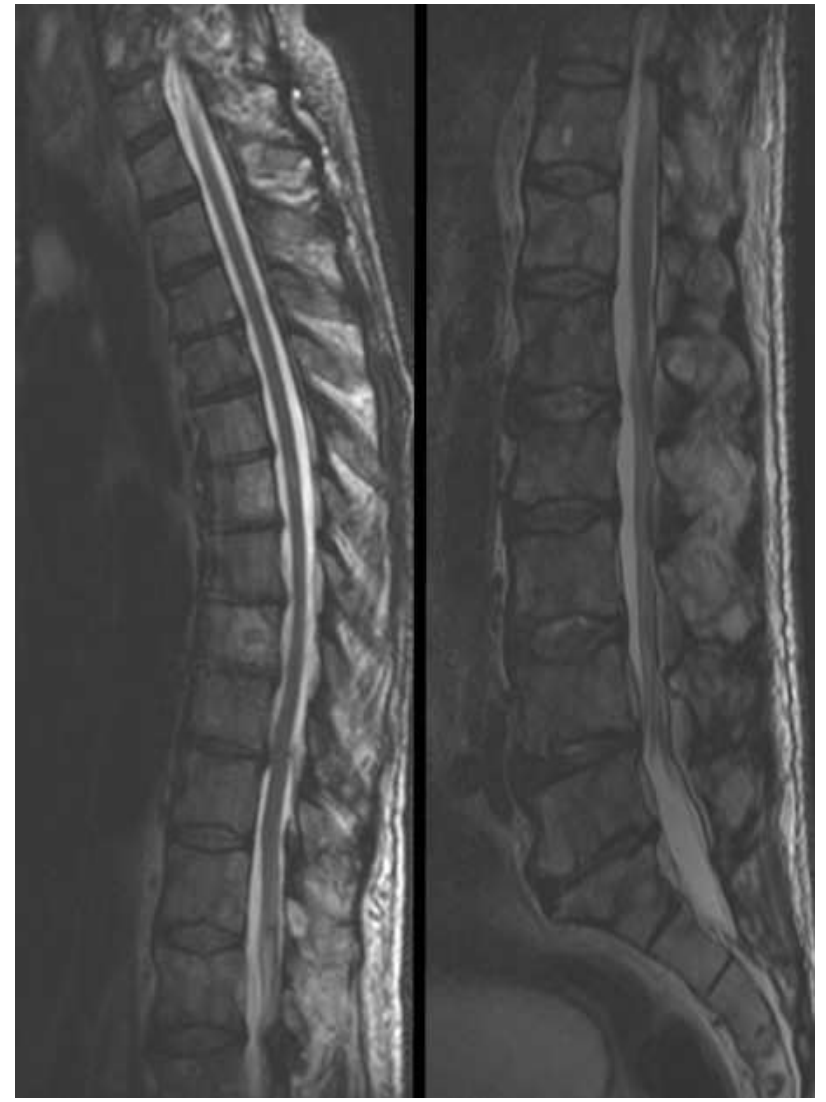

Figure 3: MRI scan of the entire spine without contrast shows vertebral bony lesions with no cord compression or fracture.

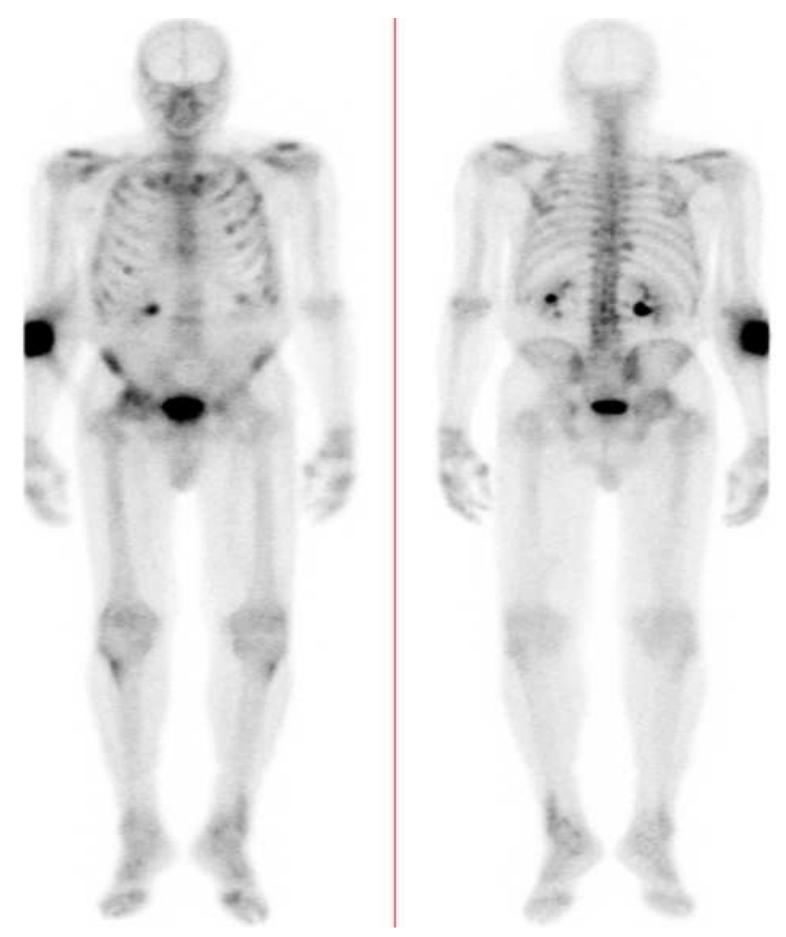

Figure 4: Technetium-bone scan shows diffuse reduce uptake (cold spots) involving several ribs and vertebrae interspersed with mild irregular uptake suggestive of predominantly diffuse osteolytic activity (right forearm is injection site).

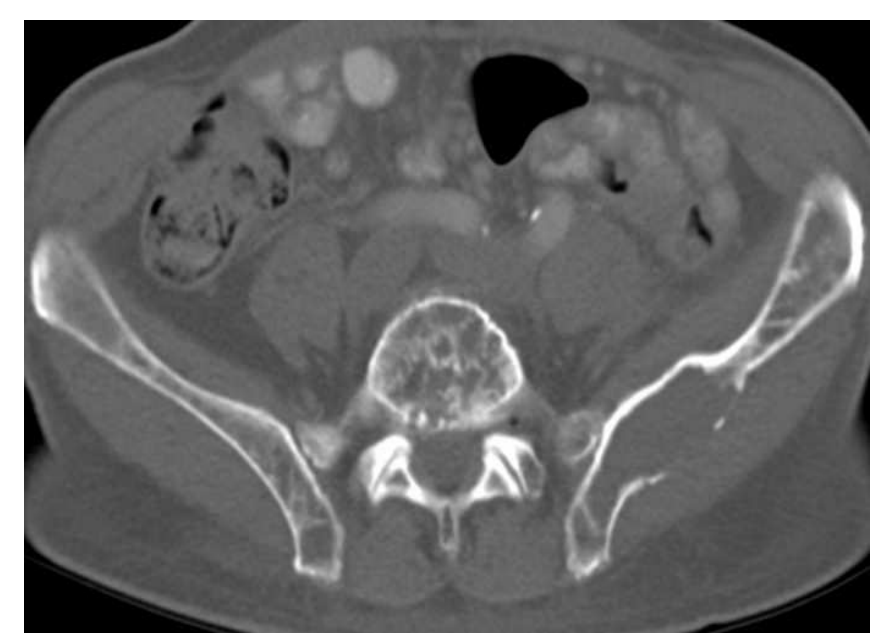

Figure 5: Post-treatment CT scan of the pelvis shows significant partial resolution of left iliac bone lesion.

of osteolytic metastases from prostate cancer and osteolytic lesions have strong resorptive component which increases the propensity for skeletal related events $[5,6,7]$. Our patient had diffuse osteolytic metastases in the pelvis and spine but he did not develop disabling skeletal complications and he responded well to $\mathrm{ZA}$, hormonal and palliative radiation therapy. Post-treatment imaging studies confirmed significant regression of osteolytic lesions with no new metastatic bone lesions.

Recent research studies have focused on the pathogenesis of bone metastasis to identify potential targets for therapeutic intervention. Metastatic tumor cells in the bone dysregulate the coordinated relationship between osteoclastic and osteoblastic functions and promote tumor cell proliferation and bone destruction with the aid of various cytokines and growth factors $[8,9]$. The pathologic dominance determines the common radiographic patterns of bone lesions that vary from predominantly osteolytic, as commonly seen in breast cancer and multiple myeloma, to predominantly osteoblastic, as commonly seen in prostate cancer $[3,4,8,9]$. Research studies have demonstrated that the markers of bone resorption are elevated to a greater degree in patients with bone metastases from prostate cancer than bone metastases from any other malignancies. The expression of IL-1, IL-6, RANKL (receptor activator of nuclear factor $\kappa B$ ligand) and parathyroid hormone-related peptide by prostate cancer cells promote osteoclastogenesis. The traid of RANKL /RANK/ osteoprotegerin has been identified as a key regulator of bone resportion. RANKL expressed on marrow stromal cells and osteoblasts is a potent inducer of osteoclast formation, and its binding to RANK receptors on osteoclast precursors trigger differentiation and activation. This process is controlled by osteoprotegerin, a decoy receptor for RANKL, which inhibits osteoclast differentiation and 
resorption. The presence of osteoclast-stimulating cytokines such as IL-6, IL-1, IL-17 and colonystimulating factors in the bone microenvironment also induces osteoclast function [9]. The release of growth factors (like TGF- $\beta$ ) secondary to bone lysis promotes cancer cell growth and endures a vicious cycle of tumor growth and bone destruction. We speculate that our patient had predominant pathologic osteoclast activity in the metastatic bone lesions resulting in osteolytic radiographic appearance.

Bone is a common site for metastasis in patients with prostate adenocarcinoma and results in significant disabling complications like pathological fracture, spinal cord compression, severe bone pain and impaired mobility, unless intervened [9]. Despite all the novel diagnostic and therapeutic methods, advanced prostate cancer disease remains incurable, and the median survival between the diagnosis of a clinically evident metastasis in bones and death is approximately $3-5$ years [8]. Treatment is mainly palliative and supportive therapy plays an important role in the prevention of skeletal complications and reducing bone pain in these patients. A range of options are available for treating skeletal metastases, including antineoplastic therapies that are directed at the tumor cells and other supportive or palliative therapies aimed at attenuating the skeletal complications, including bisphosphonates, radiotherapy, radiopharmaceuticals, surgery, vertebroplasty and kyphoplasty [4].

Zoledronic acid, an aminobisphosphonate, exerts potent inhibition of osteoclast adherence or activity and enhances osteoclast apoptosis; and it is currently used to prevent skeletal complications from bone metastases [3, 4, 9]. The NCCN (National Comprehensive Cancer Network) recommends the use of ZA every three to four weeks in men with castrateresistant prostate cancer and bone metastases. Other bisphosphonates like pamidronate and clodronate did not demonstrate an overall treatment benefit relative to placebo in these patients with advanced disease [4]. ZA (4 mg intravenously every three to four weeks) was approved by United States Food and Drug Administration (US FDA) to treat men with prostate cancer metastatic to bone and with disease progression despite first-line hormone therapy, whereas outside the US, ZA is approved for use in patients with bone metastasis from prostate cancer regardless of their hormone responsive status [4]. Randomized trial showed patients treated with ZA experienced fewer SREs (33\% Vs 44\%; $\mathrm{p}=0.021$ ) and a prolonged time interval to the occurrence of the first SRE (not reached vs. 321 days; $p=0.011$ ). Extended study confirmed the initial data and showed that the treatment group achieved a significant reduction in the incidence of skeletal complications ( $38 \%$ vs. $49 \%$; $p=0.028$ ) $[4,9]$. Due to the presence of extensive osteolytic metastases in our patient, he was started on zoledronic acid along with ADT to prevent disabling skeletal complications.

This case emphasizes the importance of regular screening for prostate cancer. The 5-year relative survival among men with localized prostate cancer or with just regional spread is $100 \%$, compared with $31.9 \%$ among those diagnosed with distant metastases [1]. Although the mortality risk reduction with prostate cancer screening is small and controversial, the early detection and treatment can reduce morbidity and prevent skeletal complications which adversely affect the quality of life. The present guidelines emphasize the need for men to have sufficient information regarding the risks and benefits of screening and treatment to make an informed and shared decision [2].

For men who decide to be screened, it is suggested to begin at age 50 in average risk white men and at age 40-45 in black men, men with a positive family history, and men who are known or likely to have BRCA1 mutation (Grade 2B). The screening is recommended to be continued until age (75 years) or

comorbidites limit life expectancy to less than 10 years or the patient decides against further screening (Grade 2B). Stopping screening at age 65 may be appropriate if the PSA level is less than $1.0 \mathrm{ng} / \mathrm{mL}$ [2].

\section{CONCLUSION}

Early detection and supportive treatment plays an integral role in reducing morbidity and preventing skeletal complications associated with bone metastases from prostate cancer. To our knowledge, this is the first reported case of prostate adenocarcinoma presenting with very high levels of PSA and extensive osteolytic metastases.

$* * * * * * * * *$

\section{Author Contributions}

Govarthanan Rajendiran - Substantial contributions to conception and design, Acquisition of data, Analysis and interpretation of data, Drafting the article, Revising it critically for important intellectual content, Final approval of the version to be published

Linda Green - Substantial contributions to conception and design, Acquisition of data, Analysis and interpretation of data, Drafting the article, Revising it critically for important intellectual content, Final approval of the version to be published

Gurdeep Chhabra - Substantial contributions to conception and design, Acquisition of data, Analysis and interpretation of data, Drafting the article, Revising it critically for important intellectual content, Final approval of the version to be published

\section{Guarantor}

The corresponding author is the guarantor of submission.

\section{Conflict of Interest}

Gurdeep Chhabra, Hemato-oncologist, is a speaker for 
Novartis. Other authors have no financial interests or conflict of interests.

\section{Copyright}

(C) Govarthanan Rajendiran et al. 2011; This article is distributed under the terms of Creative Commons attribution 3.0 License which permits unrestricted use, distribution and reproduction in any means provided the original authors and original publisher are properly credited. (Please see www.ijcasereportsandimages.com /copyright-policy.php for more information.)

\section{REFERENCES}

1. Ries, LAG, Melbert, D, Krapcho, M, et al (Eds). SEER Cancer Statistics Review, 1975-2004, National Cancer Institute, Bethesda, MD 2007. Available at http://seer.cancer.gov/csr/1975_2004/, based on November 2006 SEER data submission.

2. Hoffman RM. Screening for prostate cancer. Uptodate v18.3. Available at http://www.uptodate.com/contents/screening-forprostate-cancer.

3. Mundy GR. Metastasis to bone: Causes, consequences, and therapeutic opportunities. Nat Rev Cancer. 2002;2:584-93.

4. Smith MR, Brown GA, Saad F. New opportunities in the management of prostate cancer-related bone complications. Urol Oncol - Semin Ori. 2009;27(suppl):1s-20s.

5. Migita T, Maeda K, Ogata N. A case of prostate cancer associated with osteolytic bone metastases. Hinyokika Kiyo. 1999 May;45(5):371-4.

6. Fukuoka $\mathrm{H}$, Ishibashi $\mathrm{Y}$, Masuda $\mathrm{M}$ et al. A case of prostatic carcinoma with osteolytic bone metastases. Hinyokika Kiyo. 1988 Oct;34(10):1805-9.

7. Ansari MS, Nabi G, Aron M. Solitary radial head metastasis with wrist drop: a rare presentation of metastatic prostate cancer. Urol Int. 2003;70(1):779

8. Msaouel P, Pissimissis N, Halapas A, Koutsilieris M. Mechanisms of bone metastasis in prostate cancer: clinical implications. Best Pract Res Clin Endocrinol Metab. 2008 Apr;22(2):341-55

9. Saad F, Gleason DM, Murray R et al. A randomized, placebo-controlled trial of zoledronic acid in patients with hormone-refractory metastatic prostate carcinoma. J natl Cancer Inst. 2002;94:1458-68. 